\title{
Foveal structure during the induction phase of anti-vascular endothelial growth factor therapy for occult choroidal neovascularization in age- related macular degeneration
}

\author{
This article was published in the following Dove Press journal: \\ Clinical Ophthalmology \\ 3 November 2015 \\ Number of times this article has been viewed
}

\section{Mariko Kano \\ Tetsuju Sekiryu \\ Yukinori Sugano \\ Yasuharu Oguchi \\ Akira Ojima \\ Kanako Itagaki \\ Masaaki Saito}

Department of Ophthalmology, Fukushima Medical University School of Medicine, Hikarigaoka, Fukushima, Japan
Correspondence: Tetsuju Sekiryu Department of Ophthalmology, Fukushima Medical University School of Medicine, I Hikarigaoka, Fukushima 960-I295, Japan

Tel +8I 24547 I I I I

Fax +8I 245482640

Email sekiryu@fmu.ac.jp
Purpose: To evaluate the efficacy of monthly injections of aflibercept and ranibizumab on foveal structure after three months, for the treatment of occult choroidal neovascularization (CNV) in age-related macular degeneration (AMD).

Methods: We retrospectively studied 103 eyes with treatment-naïve neovascular AMD with occult and no classic CNV. Seventy-four of 103 eyes were treated with ranibizumab (intravitreal ranibizumab injection [IVR] group); 29 eyes were treated with aflibercept (intravitreal aflibercept injection [IAI] group). The best-corrected visual acuity and the retinal and choroidal structure at the fovea were evaluated using optical coherence tomography.

Results: The total foveal thickness, the height of serous retinal detachments, and subfoveal choroidal thickness were compared with baseline, and the incidence of retinal pigment epithelial elevation significantly decreased in the IAI group compared with the IVR group. In contrast, the thickness of the sensory retina at the fovea significantly decreased in the IVR group when compared with the IAI group. The logarithm of the minimum angle of resolution (logMAR) best-corrected visual acuity improved more significantly in the IVR group $(-0.085 \pm 0.164)$ than in the IAI group $(-0.020 \pm 0.125)$ at 3 months $(P=0.017)$.

Conclusion: After intravitreal injection, aflibercept more rapidly reduced subretinal fluid and subfoveal choroidal thickness. In contrast, ranibizumab decreased the sensory retinal thickness compared with aflibercept. The responses of the retinal and choroidal tissue to these anti-VEGF agents may be different during the induction phase for eyes with occult CNV secondary to neovascular AMD.

Keywords: neovascular age-related macular degeneration, occult, ranibizumab, aflibercept, retinal thickness, outer nuclear layer

\section{Introduction}

Age-related macular degeneration (AMD) is the most common cause of irreversible blindness in the industrialized world. ${ }^{1}$ Emerging anti-vascular endothelial growth factor (VEGF) therapies are considered the standard of care for neovascular AMD. AntiVEGF agents inhibit angiogenesis in the eye by suppressing abnormal blood vessel growth. ${ }^{2}$ Two landmark clinical trials using intravitreal injections of the humanized monoclonal antibody fragment ranibizumab (Lucentis; Genentech Inc., South San Francisco, CA, USA) in eyes with new-onset neovascular AMD showed that over 90\% of the treated eyes lost fewer than 15 letters of vision after 24 months of follow-up, and the visual acuity (VA) improved in $33.3 \%-40 \%$ of the patients. ${ }^{3,4}$ Aflibercept showed a similar or superior effect on neovascular AMD. ${ }^{5,6}$ 
Ranibizumab is a Fab fragment that neutralizes all active forms of VEGF-A and does not possess the fragment crystallizable (Fc) domain. Aflibercept is a decoy receptor that is constructed by fusion of the second binding domain of the VEGFR-1 and the third binding domain of the VEGFR-2 to the crystallizable portion of immunoglobulin $\mathrm{G}$ (IgG) $1 .{ }^{6,7}$ Although there are definite biological differences between ranibizumab and aflibercept, the efficacies of the two antiVEGF drugs in clinical practice are still inconclusive.

Spectral-domain optical coherence tomography (SDOCT) provides a detailed anatomical assessment of the retinal layers, especially the photoreceptor layer and pigment epithelium. Moreover, a relatively new technique using SD-OCT, the enhanced depth-imaging technique, allows evaluation of the choroidal morphology. ${ }^{8}$ The retinal and choroidal thicknesses significantly decreased after intravitreal injections of ranibizumab or aflibercept. ${ }^{9-12}$ Regarding the choroidal thickness, previous studies suggested that a decrease of choroidal thickness might be more remarkable in the eyes treated with aflibercept. ${ }^{10,11}$ Microstructural changes in retina and choroid may differ between the anti-VEGF drugs. To our knowledge, the morphological difference between the eyes treated with ranibizumab and that with aflibercept has not been reported.

In this retrospective study, we compared the effects of ranibizumab and aflibercept on retinal and choroidal morphology during three consecutive monthly injections to treat choroidal neovascularization (CNV) secondary to AMD in the induction phase in humans. CNV with predominantly or minimally classic lesion is associated with less improvement in VA at anti-VEGF therapies in the multicenter study. ${ }^{13}$ The integrity of the retinal structure after treatment of neovascular AMD also varied among the CNV subtypes. ${ }^{14}$ We thought that occult CNV may be suitable to analyze the retinal structure changes because the location of CNV had little influence on the foveal retinal structure. In the current study, we therefore focused on eyes with pure occult CNV, that is, CNV with no classic lesions, to minimize the variations among CNV subtypes.

\section{Methods}

This was a retrospective interventional study of treatmentnaïve patients who had occult $\mathrm{CNV}$ with no classic lesions secondary to neovascular AMD. After the potential risk and benefits were explained in detail, all patients provided written informed consent approved by Institutional Review Board at Fukushima Medical University. We analyzed the medical records of 103 eyes of 100 patients examined at Fukushima Medical University between March 2009 and December
2013. All patients underwent measurement of best-corrected visual acuity (BCVA), slit lamp biomicroscopy, color fundus photography, and OCT at baseline and 1 month, 2 months, and 3 months. The records of the eyes were discarded when there were no good fundus photographs or OCT images for analysis. The 74 eyes of 71 patients who visited from March 2009 to November 2012 were treated with a monthly intravitreal ranibizumab ( $0.5 \mathrm{mg}$ ) injection (IVR) for 3 months. The 29 eyes of 29 patients who visited from December 2012 to December 2013, aflibercept became available for medical use in Japan after December 2012, were treated with monthly intravitreal aflibercept ( $2 \mathrm{mg}$ ) injection (IAI) for 3 months. All treatment-naïve patients were treated within 3 months from onset.

The diagnosis of occult neovascular AMD with no classic CNV was based on funduscopy, angiography, and OCT findings. We used the definitions described in a previous multicenter study as the fluorescein angiographic criteria for occult CNV. ${ }^{15}$ To discriminate polypoidal choroidal vasculopathy from occult $\mathrm{CNV}$, indocyanine green angiography (ICGA) was performed in all eyes. We used the BCVA measured with a Japanese standard decimal VA chart. The BCVA was converted to the logarithm of the minimum angle of resolution ( $\log$ MAR) units before the calculations were performed.

The inclusion criteria were: a patient age of at least 50 years with a diagnosis of primary occult subfoveal $\mathrm{CNV}$ with no classic lesions secondary to AMD and juxtafoveal lesions with leakage affecting the fovea were allowed, a total occult $\mathrm{CNV}$ lesion on fluorescein angiography $<12$ disc areas, and a BCVA of 0.05 or better using the Japanese decimal VA chart.

The exclusion criteria were any of the following in either eye: CNV secondary to other macular disorders and eyes with polypoidal choroidal vasculopathy based on ICGA findings, which showed polypoidal structures at the border of the branching choroidal vascular networks ${ }^{16}$ and subpigment epithelial orange-red protrusions seen biomicroscopically that corresponded to the polypoidal lesions observed on ICGA. Other exclusion criteria included a spherical equivalent of -6.5 diopters (D) or less and/or chorioretinal atrophic changes secondary to pathologic myopia, a history of intraocular surgery within 6 months, a history of pars plana vitrectomy, a retinal pigment epithelial (RPE) tear at the first examination, or a systemic contraindication for IVR or IAI.

\section{Measurements}

The primary study outcomes were retinal and choroidal morphologic changes after IAI and IVR. To assess the retina 
using OCT, $9 \mathrm{~mm}$ horizontal and vertical scans through the foveal center were routinely performed in all study eyes using SD-OCT (Spectralis OCT; Heidelberg Engineering, Heidelberg, Germany). Additional scans were made through lesions of interest. The subfoveal choroidal thickness (SFCT) was measured using the enhanced depth-imaging technique. ${ }^{8}$ All images were obtained using an eye-tracking system (ART, installed in Spectralis OCT), with 100 scans being averaged.

We measured the total foveal thickness (TFT), the distance from the surface of the RPE to the internal limiting membrane (ILM), the foveal retinal thickness (FRT, the distance from the ILM to the external limiting membrane [ELM]), the height of serous retinal detachments (HSRD, the distance from the ELM line to the surface of the RPE), and the SFCT (the distance between the hyperreflective line corresponding to Bruch's membrane beneath the RPE and the inner surface of the sclera at the foveal center; Figure 1). The measurement was performed using the calliper function of the OCT instrument.

The structures of the ELM line, ellipsoid zone, the incidence of serous retinal detachment (SRD), intraretinal fluid (IRF), and the RPE elevation at the fovea were qualitatively assessed. RPE elevation included RPE separation from Bruch's membrane and any type of pigment epithelial detachment that was detected at the fovea on OCT images. Regarding the line structures of the ELM or the ellipsoid zone, "disruption" was defined as complete disappearance or disruption of the line. Eyes with any lines or zones that were identified regardless of whether they were or were not clear did not meet the definition of disruption.

Two retinal specialists ( $\mathrm{MK}$ and $\mathrm{KI}$ ) who were masked to the patients' information performed all quantitative and qualitative measurements. Regarding quantitative data,

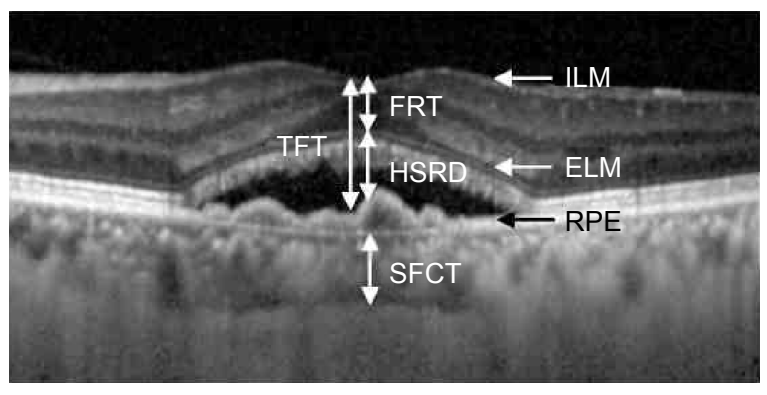

Figure I Diagram of the structures measured.

Notes: The TFT is the distance between the ILM and the inner border of the RPE. The FRT is the distance between the ILM and the ELM. The HSRD is the distance between the ellipsoid zone and the RPE. The SFCT is the distance between the outer border of the RPE and the inner scleral border.

Abbreviations: TFT, total foveal thickness; ILM, internal limiting membrane; RPE, retinal pigment epithelium; FRT, foveal retinal thickness; ELM, external limiting membrane; HSRD, height of serous retinal detachment; SFCT, subfoveal choroidal thickness. the mean value of the two graders was used for statistical analysis. Regarding qualitative data, when there were cases with inconsistent evaluations between the two graders, a third grader (TS) made the final decision. The institutional review board of Fukushima Medical University approved the study, which was conducted according to tenets of the Declaration of Helsinki.

\section{Statistical analysis}

The data obtained from all patients were analyzed with frequency and descriptive statistics. The logMAR BCVA and the mean measurement values were compared using the MannWhitney $U$ test. Categorical data were tested using Fisher's exact test. A $P$-value $<0.05$ was considered significant. All descriptive statistics were performed using JMP software version 11.0 (SAS Institute Inc., Cary, NC, USA).

\section{Results}

No significant differences in the baseline characteristics were observed between the two groups (Table 1). No systemic events were observed in two groups. The age of the patients ranged from 53 to 93 years $(75 \pm 8$ years [mean \pm standard deviation]) in the IAI group and $76 \pm 8$ years in the IVR group $(P=0.755)$. The IAI group included 27 male and two female patients, and the IVR group included 59 male and 12 female patients $(P=0.339)$. The disc areas of the occult $\mathrm{CNV}$ seen on the fluorescein angiography images were $3.5 \pm 2.6$ in the IAI group and $4.1 \pm 2.5$ in the IVR group $(P=0.334)$. The mean logMAR BCVAs before treatment did not differ significantly $(P=0.721)$ between eyes treated with

Table I Baseline characteristics of patients in the study

\begin{tabular}{llll}
\hline & IAI & IVR & $P$-value \\
\hline No (eyes/patients) & $29 / 29$ & $74 / 71$ & \\
Female:male & $2: 27$ & $12: 59$ & 0.339 \\
Age (years) & $75 \pm 8$ & $76 \pm 8$ & 0.755 \\
logMAR BCVA & $0.292 \pm 0.309$ & $0.299 \pm 0.27 \mid$ & 0.72 I \\
Lesion size (DA) & $3.5 \pm 2.6$ & $4.1 \pm 2.5$ & 0.334 \\
TFT $(\mu \mathrm{m})$ & $249 \pm 85$ & $267 \pm 115$ & 0.634 \\
FRT $(\mu \mathrm{m})$ & $110 \pm 72$ & $120 \pm 68$ & 0.198 \\
HSRD $(\mu \mathrm{m})$ & $139 \pm 77$ & $147 \pm 110$ & 0.572 \\
SFCT $(\mu \mathrm{m})$ & $242 \pm 93$ & $239 \pm 95$ & 0.866 \\
ELM $($ disrupted) & $1(3.5 \%)$ & $5(6.8 \%)$ & 1.000 \\
Ellipsoid zone (disrupted) & $8(27.6 \%)$ & $23(30.1 \%)$ & 0.814 \\
SRD & $27(93.1 \%)$ & $62(83.7 \%)$ & 0.339 \\
RPE elevation & $25(86.1 \%)$ & $65(87.4 \%)$ & 1.000 \\
IRF & $6(20.6 \%)$ & $23(31.0 \%)$ & 0.338 \\
\hline
\end{tabular}

Note: Measured data is expressed as mean \pm standard deviation.

Abbreviations: IAI, intravitreal aflibercept injection; IVR, intravitreal ranibizumab injection; logMar BCVA, the logarithm of the minimum angle of resolution bestcorrected visual acuity; DA, disc area; TFT, total foveal thickness; FRT, foveal retinal thickness; HSRD, height of serous retinal detachment; SFCT, subfoveal choroidal thickness; ELM, external limiting membrane; SRD, serous retinal detachment; RPE, retinal pigment epithelium; IRF, intra retinal fluid. 
aflibercept and those treated with ranibizumab $(0.292 \pm 0.309$ and $0.299 \pm 0.271$, respectively). There were no significant differences in the TFT $(P=0.634)$, FRT $(P=0.198)$, HSRD $(P=0.572)$, or SFCT $(P=0.866$; Table 1$)$. The incidence rates of an abnormality in the layer structure, disruption of the $\operatorname{ELM}(P=1.000)$, disruption or absence of the ellipsoid zone ( $P=0.814)$, SRD $(P=0.339)$, or RPE elevation $(P=1.000)$ did not differ between the two groups.

The changes in each measured value are shown in Table 2 and Figures 2-6. The mean logMAR BCVA in the IVR group improved consecutively at 1 month, 2 months, and 3 months; those in the IAI group decreased at 1 month and improved at 2 months and 3 months. Significant differences between the two groups were seen at each time point, $P=0.002, P=0.017$, and $P=0.017$, respectively. To determine the visual prognosis, we investigated the BCVA changes at 6 months from the clinical records, although the treatment regimens differed. The IVR group received additional injections according to the criteria during the second year of the Prospective OCT Imaging of Patients with Neovascular AMD Treated with Intraocular Ranibizumab (PrONTO) study. ${ }^{17}$ The IAI group received bimonthly injections after 3 months of the loading dose. The changes in the $\log M A R$ BCVA from baseline at 6 months were $0.009 \pm 0.127(\mathrm{n}=27)$ in the IAI group and $-0.064 \pm 0.199(\mathrm{n}=71)$ in the IVR group. No significant $(P=0.281)$ difference was observed in the logMAR BCVA between the two groups at 6 months.

Table 2 Changes in the parameter values

\begin{tabular}{|c|c|c|c|c|c|c|}
\hline \multirow[t]{2}{*}{ Parameter } & \multirow[t]{2}{*}{ Month } & \multicolumn{2}{|l|}{ IAI } & \multicolumn{2}{|l|}{ IVR } & \multirow[t]{2}{*}{$P$-value } \\
\hline & & Mean & SD & Mean & SD & \\
\hline $\log M A R$ & I & 0.016 & 0.010 & -0.064 & 0.130 & 0.002 \\
\hline \multirow[t]{2}{*}{ BCVA change } & 2 & -0.012 & 0.118 & -0.084 & 0.155 & 0.017 \\
\hline & 3 & -0.020 & 0.125 & -0.085 & 0.164 & 0.017 \\
\hline TFT change & I & $-8 \mid$ & 108 & -29 & 103 & 0.002 \\
\hline \multirow[t]{2}{*}{$(\mu \mathrm{m})$} & 2 & -79 & 115 & -49 & 113 & 0.016 \\
\hline & 3 & -78 & 125 & -52 & 115 & 0.043 \\
\hline FRT change & I & -5 & 60 & -24 & 56 & 0.001 \\
\hline \multirow[t]{2}{*}{$(\mu \mathrm{m})$} & 2 & -11 & 67 & -21 & 53 & 0.009 \\
\hline & 3 & -1 & 50 & -18 & 57 & 0.001 \\
\hline HSRD change & I & -85 & 86 & -53 & 84 & 0.008 \\
\hline \multirow[t]{2}{*}{$(\mu \mathrm{m})$} & 2 & -90 & 93 & -70 & 98 & 0.059 \\
\hline & 3 & -79 & 111 & -69 & 98 & 0.129 \\
\hline SFCT change & I & -12 & 18 & -7 & 17 & 0.279 \\
\hline \multirow[t]{2}{*}{$(\mu \mathrm{m})$} & 2 & -33 & 52 & -13 & 23 & 0.003 \\
\hline & 3 & -27 & 16 & -15 & 24 & $<0.001$ \\
\hline
\end{tabular}

Abbreviations: IAI, intravitreal aflibercept injection; IVR, intravitreal ranibizumab injection; SD, standard deviation; BCVA, best-corrected visual acuity; TFT, total foveal thickness; FRT, foveal retinal thickness; HSRD, height of serous retinal detachment; SFCT, subfoveal choroidal thickness; logMAR, logarithm of the minimum angle of resolution.

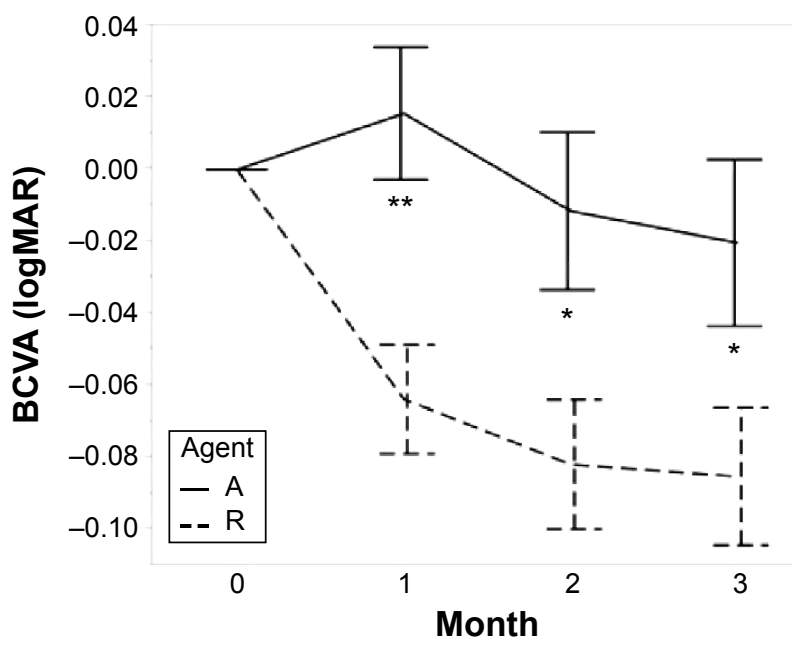

Figure 2 The graph of visual acuity.

Notes: Agent A: treated with aflibercept (IAI group). Agent R: treated with ranibizumab (IVR group). Error bars are \pm I standard error of the mean. $* P<0.05$; $* * P<0.01$.

Abbreviations: BCVA, best-corrected visual acuity; IAI, intravitreal aflibercept injection; IVR, intravitreal ranibizumab injection; logMAR, logarithm of minimal angle of resolution.

The TFT decreased from baseline after anti-VEGF therapy in both groups at each visit. The differences in the TFT changes between the two groups were significant ( 1 month, $P=0.002 ; 2$ months, $P=0.016 ; 3$ months, $P=0.043$; Table 2). The FRT also decreased in both groups, and the change was greater in the IVR group than in the IAI group ( 1 month, $P=0.001 ; 2$ months, $P=0.009 ; 3$ months, $P=0.001$; Table 2). The HSRD significantly decreased after injections of both drugs. The change in the HSRD at 1 month was

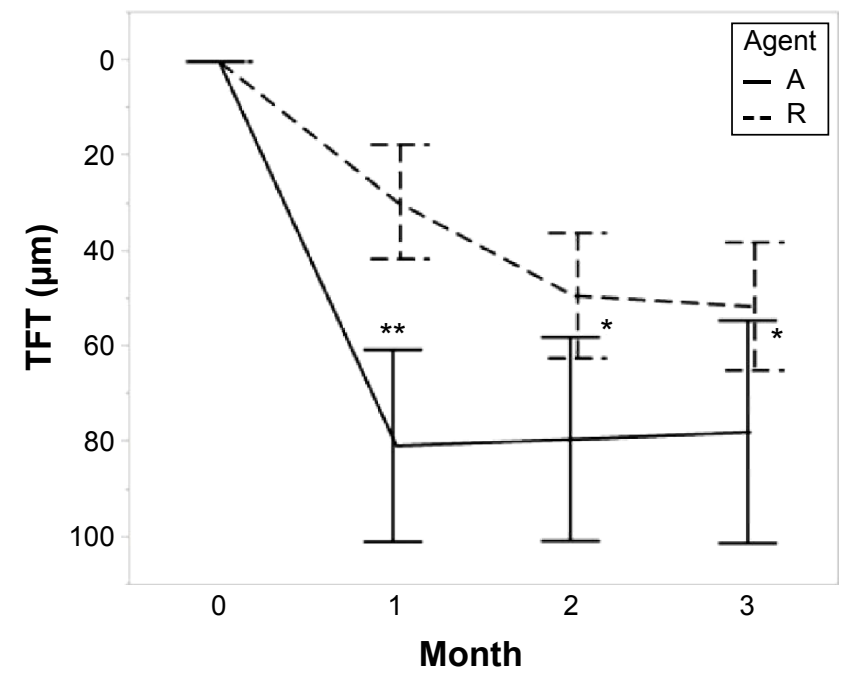

Figure 3 Total foveal thickness.

Notes: Agent A: treated with aflibercept (IAI group). Agent R: treated with ranibizumab (IVR group). Error bars are \pm I standard error of the mean. $* P<0.05$; $* * p<0.01$.

Abbreviations: IAI, intravitreal aflibercept injection; IVR, intravitreal ranibizumab injection; TFT, total foveal thickness. 


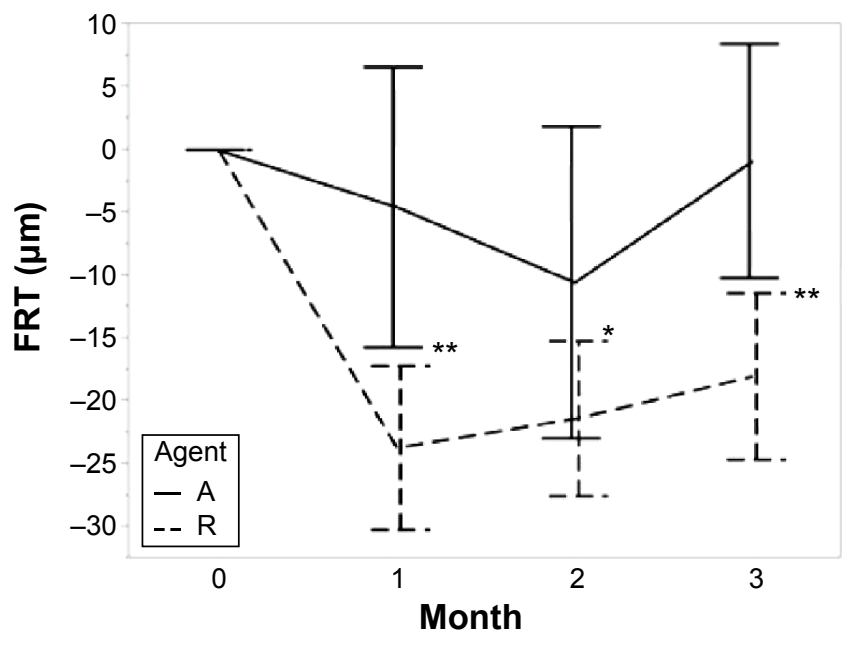

Figure 4 Foveal retinal thickness (the distance from the internal limiting membrane to the external limiting membrane).

Notes: Agent A: treated with aflibercept (IAI group). Agent R: treated with ranibizumab (IVR group). Error bars are \pm I standard error of the mean. $* P<0.05$; $* * p<0.01$.

Abbreviations: IAI, intravitreal aflibercept injection; IVR, intravitreal ranibizumab injection; FRT, foveal retinal thickness.

significantly greater $(P=0.008)$ in eyes treated with aflibercept than in those treated with ranibizumab. The differences in the changes in the HSRD between the two groups did not reach significance at 2 months and 3 months $(P=0.059$ and $P=0.129$, respectively). The SFCT gradually decreased after treatment in both groups. Although the changes in the SFCT between the two groups were not significant $(P=0.279)$ at 1 month, the SFCT in the IAI group significantly decreased at 2 months and 3 months compared with that in the IVR group ( $P=0.003$ and $P<0.001$, respectively). The rates of

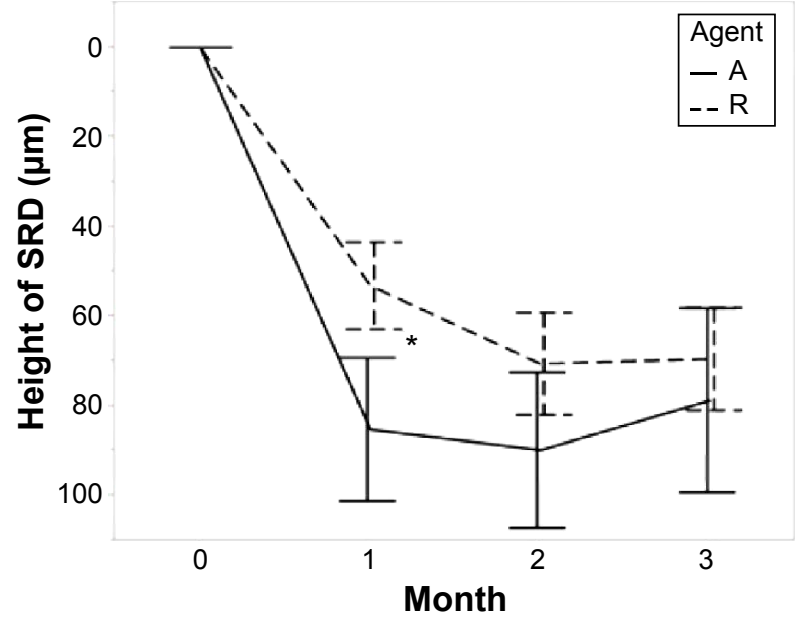

Figure 5 Height of serous retinal detachment.

Notes: Agent A: treated with aflibercept (IAI group). Agent R: treated with ranibizumab (IVR group). Error bars are \pm I standard error of the mean. $* P<0.05$. Abbreviations: IAI, intravitreal aflibercept injection; IVR, intravitreal ranibizumab injection; Height of SRD, Height of serous retinal detachment.

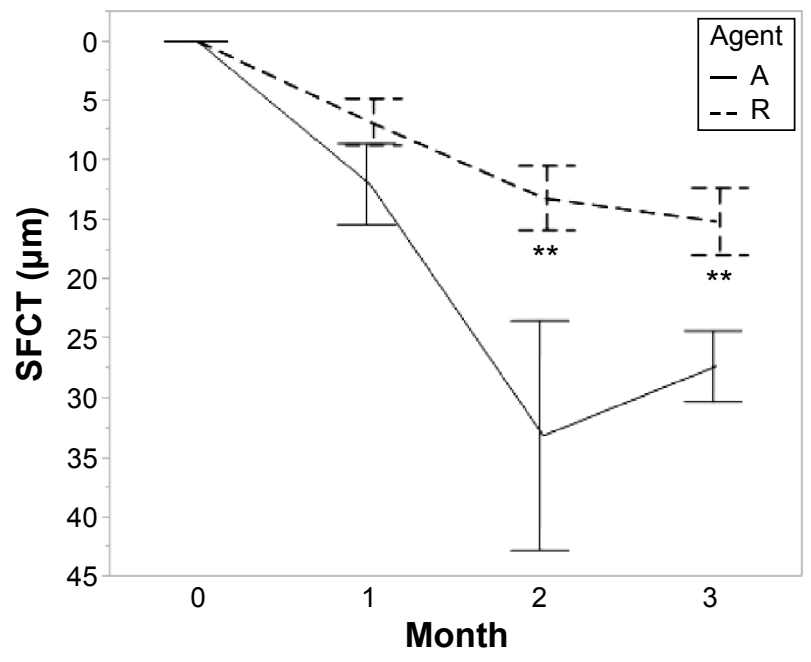

Figure 6 Subfoveal choroidal thickness.

Notes: Agent A: treated with aflibercept (IAI group). Agent R: treated with ranibizumab (IVR group). Error bars are \pm I standard error of the mean. $* * P<0.01$.

Abbreviations: IAI, intravitreal aflibercept injection; IVR, intravitreal ranibizumab injection; SFCT, subfoveal choroidal thickness.

SFCT reduction at 3 months were $11.3 \%$ in the IAI group and $6.3 \%$ in the IVR group.

Regarding qualitative parameters, the incidence rates of SRD and RPE elevation were low in eyes treated with aflibercept at each observation point after the intravitreal injections. The incidence of SRD at 3 months was significantly lower $(P=0.036)$ in eyes treated with aflibercept. The incidence rates of RPE elevation at 2 months and 3 months also were significantly lower $(P=0.008$ and $P=0.030$, respectively) in eyes treated with aflibercept compared with eyes treated with ranibizumab. The incidence of disruption of the ellipsoid zone was low in the ranibizumab group at each observation point after the intravitreal injections. The differences between the two groups did not reach significance. The incidence rates of ELM disruption and IRF did not differ between the groups (Table 3). We analyzed the factors associated with logMAR changes at 3 months using stepwise analysis. The dependent variable was the change in the logMAR BCVA at 3 months. The explanatory variables were the treatment drugs and changes in the TFT, FRT, HSRD, SFCT, ELM, ellipsoid zone, SRD, and RPE elevation at 3 months. The minimal Bayesian information criterion was used as the stopping rule. The status of the FRT and the ellipsoid zone remained as probable factors. Fitting to a least squares model using the two probable factors showed that the decrease in the FRT was the only significant factor $(P=0.012)$ correlated with the improvement of the logMAR BCVA at 3 months (Table 4). The decreasing FRT was correlated with the logMAR BCVA improvement in each group (Figure 7). 
Table 3 Incidence rates of the qualitative parameters

\begin{tabular}{|c|c|c|c|c|c|}
\hline Month & \multicolumn{2}{|c|}{ IAI $(n=29)$} & \multicolumn{2}{|c|}{ IVR $(n=74)$} & $P$-value \\
\hline \multicolumn{6}{|c|}{ ELM disruption } \\
\hline 1 & 3 & $10.3 \%$ & 4 & $5.4 \%$ & 0.079 \\
\hline 2 & I & $3.4 \%$ & 8 & $10.8 \%$ & 0.099 \\
\hline 3 & 2 & $6.9 \%$ & 9 & $12.2 \%$ & 0.036 \\
\hline \multicolumn{6}{|c|}{ Disruption of ellipsoid line } \\
\hline 1 & 12 & $72.4 \%$ & 21 & $28.4 \%$ & 0.243 \\
\hline 2 & 14 & $58.6 \%$ & 28 & $37.8 \%$ & 0.377 \\
\hline 3 & 9 & $55.2 \%$ & 20 & $27.0 \%$ & 0.808 \\
\hline \multicolumn{6}{|l|}{ SRD } \\
\hline 1 & 10 & $34.5 \%$ & $4 I$ & $55.4 \%$ & 0.079 \\
\hline 2 & 6 & $20.7 \%$ & 26 & $35.1 \%$ & 0.099 \\
\hline 3 & 5 & $17.2 \%$ & 31 & $41.9 \%$ & 0.036 \\
\hline \multicolumn{6}{|l|}{ PEE } \\
\hline I & 21 & $72.4 \%$ & 63 & $85.1 \%$ & 0.161 \\
\hline 2 & 17 & $58.6 \%$ & 62 & $83.8 \%$ & 0.008 \\
\hline 3 & 16 & $55.2 \%$ & 58 & $78.4 \%$ & 0.030 \\
\hline \multicolumn{6}{|l|}{ IRF } \\
\hline 1 & 4 & $13.8 \%$ & 13 & $17.6 \%$ & 0.773 \\
\hline 2 & 2 & $6.9 \%$ & 11 & $14.9 \%$ & 0.342 \\
\hline 3 & 4 & $13.8 \%$ & 10 & $13.5 \%$ & 1.000 \\
\hline
\end{tabular}

Abbreviations: ELM, external limiting membrane; IAl, intravitreal aflibercept injection; IVR, intravitreal ranibizumab injection; SRD, serous retinal detachment; PEE, pigment epithelial elevation; IRF, intra retinal fluid.

\section{Discussion}

The foveal structure seen on OCT images was retrospectively evaluated during the initial three monthly intravitreal injections of aflibercept or ranibizumab in eyes with occult CNV secondary to neovascular AMD. The TFT, HSRD, SFCT, and the incidence of RPE elevation significantly decreased in the IAI group. In contrast, the FRT significantly decreased in the IVR group. The ellipsoid zone was restored in the IVR group but did not reach significance. The mean $\operatorname{logMAR}$ BCVA significantly increased in the IVR group compared with the IAI group within 3 months after the intravitreal injection.

The strong effects of aflibercept for suppressing exudative changes in the retina and choroid were demonstrated in this study. The TFT markedly and rapidly decreased within 4 weeks in the VIEW study, and our results were consistent with those of this study. Because reduction of the HSRD showed a similar pattern to that of the TFT, the HSRD reduction accounted for the decrease in the TFT. The reduction rate $(6.3 \%)$ of the SFCT at 3 months in the IVR group was similar to those reported previously, $1.3 \%-7.4 \% .{ }^{9}, 10$ The reduction rate of the SFCT in the IAI group was greater than that in the IVR group. This finding is consistent with the previous report in which the SFCT in the eyes treated with ranibizumab decreased after switching the treatment from ranibizumab to aflibercept. ${ }^{18}$ Experimentally, the reductions in the density of the choriocapillaris and number of endothelial cell fenestrations in the choriocapillaris were decreased in monkey eyes treated with aflibercept compared with those treated with ranibizumab. ${ }^{19}$ The suppression of permeability in the choroid or subpigment epithelial neovascular tissue may be attributed to the SFCT reduction and resolution of subretinal fluid.

Although eyes treated with aflibercept showed rapid resolution of subretinal fluid, the logMAR BCVA decreased at 1 month in the IAI group. Temporary decreases or fluctuations of the BCVA during the initial three monthly injections of aflibercept for the eyes previously treated with ranibizumab have been recently reported. ${ }^{18,20}$ Among the morphologic parameters, thinning of the sensory retina was correlated with visual improvement. It was reported that the reduction of sensory retinal thickness was related to visual improvement after anti-VEGF therapy, ${ }^{21}$ so our results were consistent with those of the previous reports.

There are several possibilities why the FRT of the eyes treated with aflibercept did not decrease as much as those treated with ranibizumab. The condition of the retina at baseline may be different between two groups. FRT value was $110 \pm 72 \mu \mathrm{m}$ in aflibercept and $120 \pm 68 \mu \mathrm{m}$ in ranibizumab at baseline. The difference could affect the FRT change. However, FRT value was $109 \pm 33 \mu \mathrm{m}$ in aflibercept and $102 \pm 40 \mu \mathrm{m}$ in ranibizumab at 3 months $(P=0.259)$. FRT in IAI group hardly changed for 3 months, whereas it remarkably decreased in IVR group and was thinner than that in

Table 4 Predictor of visual improvement at 3 months (least squares model)

\begin{tabular}{|c|c|c|c|c|}
\hline & Estimate & Standard error & $T$ ratio & $P$-value \\
\hline \multicolumn{5}{|l|}{ FRT change at 3 months } \\
\hline FRT at 3 months - FRT at baseline & 0.000699 & 0.000273 & -2.56 & 0.012 \\
\hline \multicolumn{5}{|l|}{ Ellipsoid line } \\
\hline Not disrupted $=0$ & 0.066031 & 0.033504 & 1.97 & 0.0515 \\
\hline \multicolumn{5}{|l|}{ Disrupted $=1$} \\
\hline Intercept & -0.105175 & 0.029131 & -3.61 & 0.0005 \\
\hline
\end{tabular}

Notes: Fitting to the least squares model using the two probable factors. Dependent variable: visual improvement at 3 months. Abbreviation: FRT, foveal retinal thickness. 


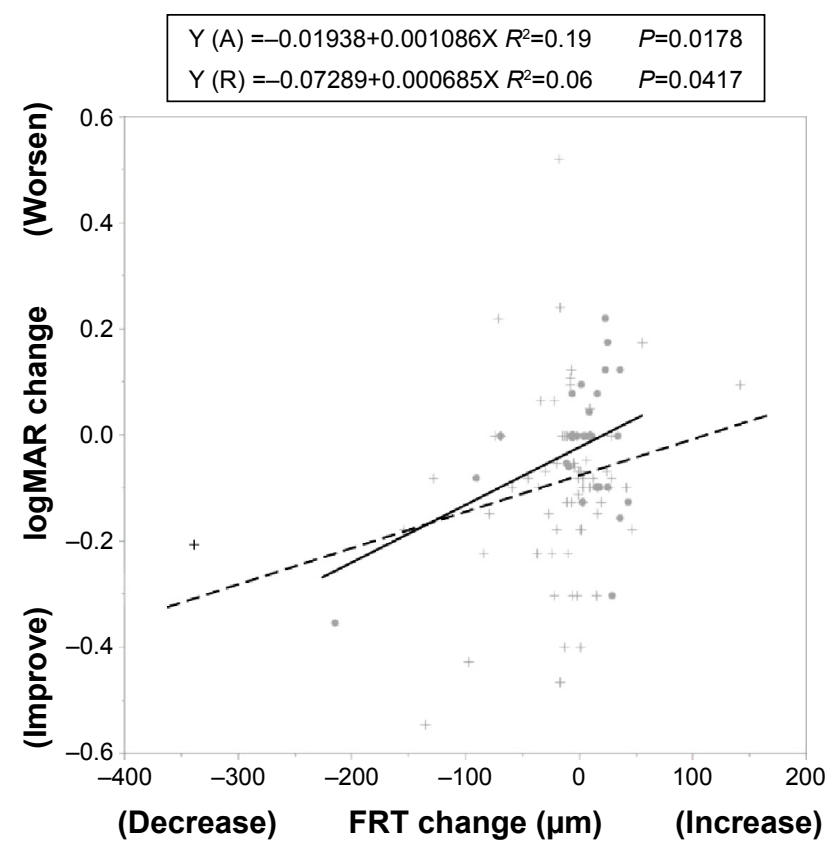

Figure 7 The visual acuity and the thickness of neurosensory retina.

Notes: FRT change $=$ FRT at 3 months - FRT at baseline; logMAR BCVA change $=\log M A R B C V A$ at 3 months $-\log$ MAR BCVA at baseline. $\bullet$ aflibercept (A), + ranibizumab ( $R)$.

Abbreviations: FRT, fovea retinal thickness; logMAR BCVA, the logarithm of the minimum angle of resolution best-corrected visual acuity.

IAI group at 3 months. We think that the FRT change might not be influenced by the baseline condition of the retina between two groups.

The difference in FRT change between two drugs is attributed to swelling of the photoreceptor cells due to the ischemic status of the choroid. After photodynamic therapy, choroidal ischemia induced retinal thickening. ${ }^{22}$ Bevacizumab, ranibizumab, and aflibercept affected the endothelium of the choriocapillaris, which resulted in thrombosis of the choroidal vessels. ${ }^{23,24}$ Because aflibercept has a higher affinity for VEGF than ranibizumab, the combined high affinity and longer half-life have led to a calculated duration of effect of intravitreal injection of $2 \mathrm{mg}$ of aflibercept for $48-83$ days. ${ }^{25}$ In addition, aflibercept reduced the intraocular concentration of VEGF to extremely low levels ${ }^{26}$ after intravitreal injection in humans. Based upon these reports, it is observed that aflibercept may cause choroidal ischemia. As a result, the sensory retinal thickness may be thicker in the aflibercept group than in the ranibizumab group. Furthermore, thinning of the choroidal thickness might be involved in circulatory changes of the choroid. However, a recent study reported that the retinal microstructure in healthy primate models did not change remarkably after aflibercept injection. ${ }^{19} \mathrm{~A}$ therapeutic dose of aflibercept injection in human eyes is therefore less likely to be associated with increasing FRT.
Another possibility of the difference in FRT change is immune complex inflammation through interaction of IgG with the Fcy receptor. Aflibercept is constructed by fusion of the binding domains of the VEGFRs to the crystallizable portion of $\operatorname{IgG} 1,{ }^{6,7}$ whereas ranibizumab is a monoclonal antibody fragment (Fab) without the crystallizable (Fc) domain. ${ }^{2}$ Aflibercept was assumed to bind to vascular endothelial cells with the endothelial Fc receptor through formation of a protein complex. A recent report suggested that interaction of $\operatorname{IgG}$ with the Fc $\gamma$ receptor possibly induced immune complex inflammation, in which microglia and macrophages were activated in the retina. ${ }^{27}$ An immune reaction involving the Fc $\gamma$ receptor could be attributed to the increasing FRT in the aflibercept group. In an extended study, the change in BCVA did not differ significantly between the two groups 6 months after the intravitreal injections, and the BCVA in a longitudinal multicenter study showed that aflibercept was not inferior to ranibizumab. ${ }^{5}$ Immune reactions in the early phase might resolve when VEGF concentration decreased after serial injection of aflibercept. As we did not evaluate the parameter regarding inflammation in the current study, further studies are needed to confirm this possibility. Placental growth factor (PlGF) may have a protective effect on the retinal cells. ${ }^{28}$ Blockade of PlGF may be responsible for the change in the FRT.

The current study was limited because of its retrospective nature. All patients were Japanese, and they clinically and genetically differed from Caucasian and black patients, so racial differences may have affected the results.

\section{Conclusion}

In conclusion, after intravitreal injection, aflibercept more rapidly reduced subretinal fluid and SFCT. In contrast, ranibizumab decreased the sensory retinal thickness compared with aflibercept. The responses of the retinal and choroidal tissues to these anti-VEGF agents may be different during the induction phase for eyes with occult CNV secondary to neovascular AMD. Based upon these results, long-term investigations of the efficacies of these two treatments on retinal and choroidal tissue are needed in future studies.

\section{Disclosure}

The authors report no conflicts of interest in this work.

\section{References}

1. Wong TY, Chakravarthy U, Klein R, et al. The natural history and prognosis of neovascular age-related macular degeneration: a systematic review of the literature and meta-analysis. Ophthalmology. 2008;115(9): $116-126$. 
2. Ferrara N. The role of vascular endothelial growth factor in pathological angiogenesis. Breast Cancer Res Treat. 1995;36(2):127-137.

3. Brown DM, Kaiser PK, Michels M, et al; ANCHOR Study Group. Ranibizumab versus verteporfin for neovascular age-related macular degeneration. N Engl J Med. 2006;355(14):1432-1444.

4. Rosenfeld PJ, Brown DM, Heier JS, et al; MARINA Study Group. Ranibizumab for neovascular age-related macular degeneration. NEngl J Med. 2006;355(14):1419-1431.

5. Heier JS, Brown DM, Chong V, et al; VIEW 1 and VIEW 2 Study Groups. Intravitreal aflibercept (VEGF trap-eye) in wet age-related macular degeneration. Ophthalmology. 2012;119(12):2537-2548.

6. Holash J, Davis S, Papadopoulos N, et al. VEGF-trap: a VEGF blocker with potent antitumor effects. Proc Natl Acad Sci U S A. 2002;99(17): 11393-11398

7. Economides AN, Carpenter LR, Rudge JS, et al. Cytokine traps: multicomponent, high-affinity blockers of cytokine action. Nat Med. 2003; 9(1):47-52.

8. Margolis R, Spaide RF. A pilot study of enhanced depth imaging optical coherence tomography of the choroid in normal eyes. Am J Ophthalmol. 2009;147(5):811-815.

9. Ogasawara M, Maruko I, Sugano Y, Ojima A, Sekiryu T, Iida T. Retinal and choroidal thickness changes following intravitreal ranibizumab injection for exudative age-related macular degeneration. Nihon Ganka Gakkai Zasshi. 2012;116(7):643-649.

10. Yamazaki T, Koizumi H, Yamagishi T, Kinoshita S. Subfoveal choroidal thickness after ranibizumab therapy for neovascular age-related macular degeneration: 12-month results. Ophthalmology. 2012;119(8): $1621-1627$.

11. Koizumi H, Kano M, Yamamoto A, et al. Aflibercept therapy for polypoidal choroidal vasculopathy: short-term results of a multicentre study. Br J Ophthalmol. 2015;99(9):1284-1288.

12. Mazaraki K, Fassnacht-Riederle H, Blum R, Becker M, Michels S. Change in choroidal thickness after intravitreal aflibercept in pretreated and treatment-naïve eyes for neavascular age-related macular degeneration. Br J Ophthalmol. Epub 2015.

13. Ying GS, Huang J, Maquire MG, et al; Comparison of Age-related Macular Degeneration Treatments Trials Research Group. Baseline predictors for one-year visual outcomes with ranibizumab or bevacizumab for neovascular age-related macular degeneration. Ophthalmology. 2013;120(1):122-129.

14. Hayashi H, Yamashiro K, Tsujikawa A, Ota M, Otani A, Yoshimura N. Association between foveal photoreceptor integrity and visual outcome in neovascular age-related macular degeneration. Am J Ophthalmol. 2009; 148(1):83-89.e1.

15. Photodynamic therapy of subfoveal choroidal neovascularization in age-related macular degeneration with verteporfin: one-year results of 2 randomized clinical trials - TAP report. Treatment of age-related macular degeneration with photodynamic therapy (TAP) Study Group. Arch Ophthalmol. 1999;117(10):1329-1345.
16. Spaide RF, Yannuzzi LA, Slakter JS, Sorenson J, Orlach DA. Indocyanine green videoangiography of idiopathic polypoidal choroidal vasculopathy. Retina. 1995;15(2):100-110.

17. Lalwani GA, Rosenfeld PJ, Fung AE, et al. A variable-dosing regimen with intravitreal ranibizumab for neovascular age-related macular degeneration: year 2 of the PrONTO study. Am J Ophthalmol. 2009; 148(1):43-58.e1.

18. Gharbiya M, Iannetti L. Visual and anatomical outcomes of intravitreal aflibercept for treatment-resistant neovascular age-related macular degeneration. Biomed Res Int. 2014;2014:273754.

19. Julien S, Biesemeier A, Taubitz T, Schraermeyer U. Different effects of intravitreally injected ranibizumab and aflibercept on retinal and choroidal tissues of monkey eyes. Br J Ophthalmol. 2014;98(6):813-825.

20. Wykoff CC, Brown DM, Maldonado ME, Croft DE. Aflibercept treatment for patients with exudative age-related macular degeneration who were incomplete responders to multiple ranibizumab injections (TURF trial). Br J Ophthalmol. 2014;98(7):951-955.

21. Keane PA, Liakopoulos S, Chang KT, et al. Relationship between optical coherence tomography retinal parameters and visual acuity in neovascular age-related macular degeneration. Ophthalmology. 2008; 115(12):2206-2214.

22. Maruko I, Iida T, Sugano Y, Ojima A, Ogasawara M, Spaide RF. Subfoveal choroidal thickness after treatment of central serous chorioretinopathy. Ophthalmology. 2010;117(9):1792-1799.

23. Schnichels S, Hagemann U, Januschowski K, et al. Comparative toxicity and proliferation testing of aflibercept, bevacizumab and ranibizumab on different ocular cells. Br J Ophthalmol. 2013;97(7):917-923.

24. Schraermeyer U, Julien S. Formation of immune complexes and thrombotic microangiopathy after intravitreal injection of bevacizumab in the primate eye. Graefes Arch Clin Exp Ophthalmol. 2012;250: 1303-1313.

25. Stewart MW, Rosenfeld PJ. Predicted biological activity of intravitreal VEGF trap. Br J Ophthalmol. 2008;92(5):667-668.

26. Wang X, Sawada T, Sawada O, Saishin Y, Liu P, Ohji M. Serum and plasma vascular endothelial growth factor concentrations before and after intravitreal injection of aflibercept or ranibizumab for age-related macular degeneration. Am J Ophthalmol. 2014;158(4):738-744.

27. Murinello S, Mullins RF, Lotery AJ, Perry VH, Teeling JL. Fcgamma receptor upregulation is associated with immune complex inflammation in the mouse retina and early age-related macular degeneration. Invest Ophthalmol Vis Sci. 2014;55(1):247-258.

28. Kernt M, Neubauer AS, Liegl RG, et al. Sorafenib prevents human retinal pigment epithelium cells from light-induced overexpression of VEGF, PDGF and PIGF. Br J Ophthalmol. 2010;94(11):1533-1539.
Clinical Ophthalmology

\section{Publish your work in this journal}

Clinical Ophthalmology is an international, peer-reviewed journal covering all subspecialties within ophthalmology. Key topics include: Optometry; Visual science; Pharmacology and drug therapy in eye diseases; Basic Sciences; Primary and Secondary eye care; Patient Safety and Quality of Care Improvements. This journal is indexed on Submit your manuscript here: http://www.dovepress.com/clinical-ophthalmology-journal

\section{Dovepress}

PubMed Central and CAS, and is the official journal of The Society of Clinical Ophthalmology (SCO). The manuscript management system is completely online and includes a very quick and fair peer-review system, which is all easy to use. Visit http://www.dovepress.com/ testimonials.php to read real quotes from published authors. 